\title{
BRCA2 C.156_157 INSALU MUTATION IN A MALE BRAZILIAN PATIENT WITH BREAST CANCER
}

Deidimar Cassia Batista Abreu¹, Edna Beatriz de Souza Alencar Paiva², Pedro Paulo Batista de Abreu¹, Vera Aparecida Saddi ${ }^{1,3}$

${ }^{1}$ Instituto Goiano e Oncologia e Hematologia - Goiânia (GO), Brazil.

${ }^{2}$ Hospital Araújo Jorge - Goiânia (GO), Brazil.

${ }^{3}$ Pontifícia Universidade Católica de Goiás - Goiânia (GO), Brazil.

Introduction: BRCA2 is a tumor suppressor gene that encodes a protein involved in the repair of DNA damage. Mutations in BRCA2 are involved in the Hereditary Breast and / or Ovarian Cancer (HBOC) Syndrome. Objective: This study aims to describe the case of a male patient with a history of HBOC presenting with the founder mutation BRCA2 c.156_157insAlu. Case Description: A 76-year-old male patient had a personal history of prostate cancer, breast cancer, and a previous resection of intestinal polyps. The patient was submitted to NGS (Next Generation Sequencing) genetic test with a panel of 35 genes associated with HBOC, presenting with negative results for pathogenic variants, probably pathogenic, and variants of uncertain significance (VUS). The family history described a daughter with breast cancer with a genetic test revealing a pathogenic variant BRCA2 c.156_157insAlu, compatible with a founding mutation of the Portuguese population. The family history of the patient was limited because he did not know his biological father or relatives of paternal origin. Discussion: The BRCA2 c.156_157insAlu variant was initially described by Teugels et al., 2005, as an Alu insertion in exon 3 of the BRCA2 gene, resulting in the loss of this exon during mRNA splicing. Exon 3 is important in the tumor suppressor function of the BRCA2 protein since it is related to its transcriptional activation domain. A regional founding effect was described for this variant in Portuguese families with HBOC. Pathogenic variants such as this impact on the patient's risk against the associated tumors. Commonly used genetic tests (NGS) do not detect large insertions and deletions. Due to the massive Portuguese immigration to Brazil, more attention should be paid to the characterization of individuals during genetic counseling, emphasizing the need to complement genetic tests in cases of Portuguese ancestry, or even in the inclusion of MLPA (Multiplex Ligation-dependent Probe Amplification), in the characterization of this Portuguese founding variant. 